\title{
Decision Support Tool for Strategic Planning in Supply Chains
}

\author{
Pedro Costa ${ }^{\mathrm{a}}$, Maria Isabel Gomes ${ }^{\mathrm{b}}$, Ana Carvalho ${ }^{\mathrm{a}^{*}}$, Ana Barbosa-Póvoa ${ }^{\mathrm{a}}$ \\ ${ }^{a}$ CEG-IST, Instituto Superior Técnico, Universidade de Lisboa, Av. Rovisco Pais, 1049- \\ 001, Lisboa, Portugal \\ ${ }^{b}$ CMA, Universidade Nova de Lisboa, Monte da Caparica, 2829-526 Caparica, \\ Portugal
}

\begin{abstract}
This paper proposes a systematic methodology for supply chain planning optimization and assessment of different scenarios. This methodology will help companies throughout the difficult process of strategic decision-making in what concerns the design of an efficient supply chain structure. The proposed methodology allows the analysis of complex systems as it integrates a decision support tool developed to agile the process of scenario comparison, when designing network structures. This tool allows practitioners to take advantage of the optimization models without the need of learning modelling languages. An example based on a real case study is presented, showing the methodology/tool applicability.
\end{abstract}

Keywords: Decision Support Tool, Supply Chains, Network Design

\section{Introduction}

The globalization growth has caused industry and organizations to function and compete on a supply chain basis. Improving supply chain performance has become a critical goal for companies to gain competitive advantages in the markets (Cai et.al, 2009). Alongside with the increased requirements in terms of supply chains strong economic performance, organizations are now held responsible for the environmental and social performance of their suppliers and partners. Achieving a sustainable supply chain has now become one of the major goals (Seuring and Muller, 2008). In order to attain such sustainability, organizations have to look at the whole products/services lifecycle. Therefore it is crucial that the complete supply chain, including the forward and reverse flows, is planned in an integrated manner in order to ensure that the entire system is sustainable (Guide and Van Wassenhove, 2009). In the above context, closedloop supply chains have become a cornerstone in companies' performance (Fleischmann et al., 2001). However, such supply chains are complex systems, which are difficult to optimise since they involve several interdependent decision variables. Companies thus require generic and systematic methodologies to help their decision processes when managing such systems, allowing them to plan effective and efficiently their supply chains. In order to achieve this goal those methodologies need to be incorporated in decision-support tools (DST), which will link the academic work to companies' problems, allowing a fast and precise analysis of supply chain structures. Within this context, the present work aims to present a systematic methodology enabling the analysis of different scenarios of supply chains structures, through the application of mathematical models, which are incorporated in a DST called VisualSC. The models are developed within mathematical language software (such as GAMS) and solved by commercial solver (as for instance, CPLEX). 


\section{Methodology}

The proposed methodology, presented in Figure 1, follows a systems approach that intends to create and assess different optimized supply chain structures in a fast an efficient way. The methodology is able to deal with direct supply chains (such as forward or reverse flows) to complex structures (such as closed-loop supply chains), which simultaneously account for forward and reverse flows). Evaluating different scenarios of complex structures is a real time consuming problem involving complex models and therefore companies are usually reluctant to spend time learning and performing such type of analysis, ending up rejecting these methodologies. In order to overcome this problem a DST called VisualSC (in detail below) has been integrated within the methodological approach described, in order to agile the scenario comparison, turning complex optimization models more easily available for practitioners' analysis. The steps of the methodology are going to be explained in a step-by-step procedure.

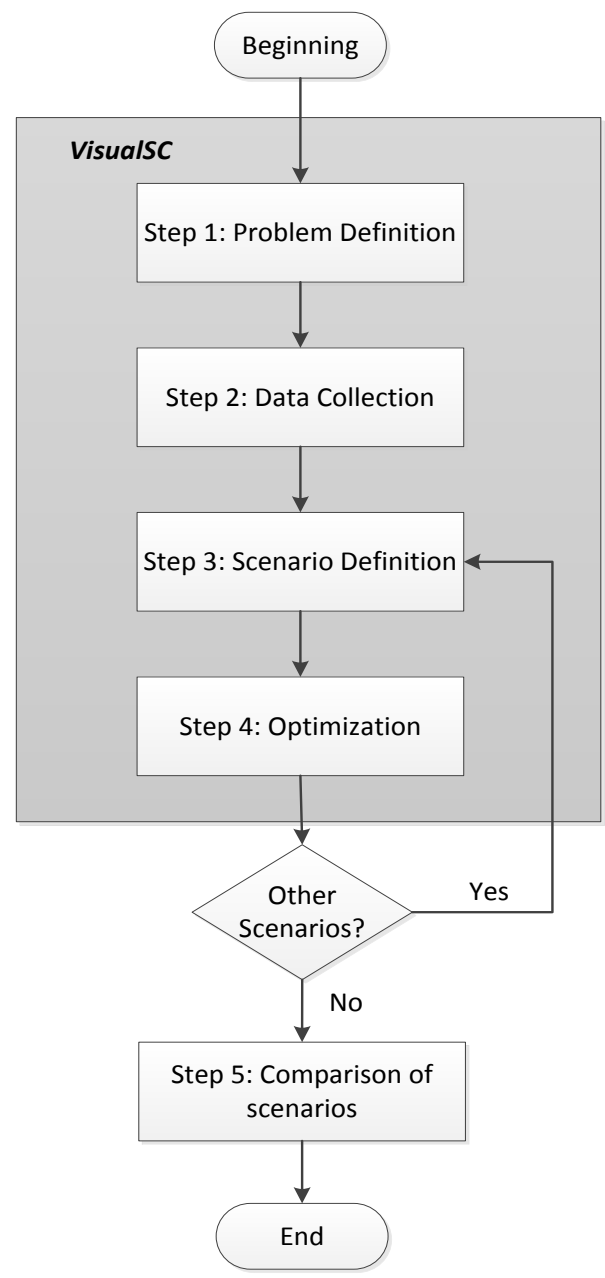

Figure 1: Methodology

\section{Step 1: Problem Definition}

In this step the type of problem in study is specified. The supply chain is characterized determining the type of flows that should be considered in the analysis (forward, reverse, closed-loop). Supply chain details, such echelons, entities (suppliers, manufacturers, warehouses, customers, etc) and their connections are also specified within this step.

\section{Step 2: Data Collection}

In step 2 data regarding the supply chain is collected. Since this is a framework for supply chain network design, the possible locations of the facilities should be defined and distances between entities should be determined. Capacities ranges are to be established for each entity. This step usually is the first bottleneck for the analysis of different scenarios in network design problems. In complex structures this task is very time consuming, since a huge amount of data is required for the analysis. In that sense this methodology takes advantage of a DST (VisualSC) where the possible entities are inserted directly through the interface and the distances between them automatically calculated by VisualSC. All data of this step is entered directly through VisualSC. The tool automatically creates a file with all the required data for the optimization model. The user does not need to understand how to work with software 
such as GAMS/CPLEX.

\section{Step 3: Scenario Definition}

In this step a scenario with its particular characteristics is specified. As example, different facility capacities might be analysed, different demand scenarios studied, different transportation modes considered or even a scenario where the network may face disruptions could be analysed. This step should be performed whenever a new scenario is required for comparison. New specifications for the supply chain are inserted in VisualSC and a new input file for GAMS is generated.

\section{Step 4: Optimization}

In step 4 the optimization model representing the supply chain should be selected. This step includes a model library where several models taking into consideration different strategic and tactical aspects of the supply chain are available. At present VisualSC incorporates a generic model that allows the modelling of different supply chains networks (forward, reverse or both forward and reverse) (Salema et al., 2010). As abovementioned, VisualSC generates a data file in step 3, which is at this step exported to GAMS enabling the model optimization. It is important to mention that this model library can easily be updated with new models. The models just need to be implemented in GAMS, since the input files generated by VisualSC are designed for GAMS software. Any strategic/tactical model, which includes supply chains networks can be added and used to assess different supply chains. Moreover, the optimization model might be a single- or a multi-objective formulation modelling, for instance, the three pillars of sustainability: economic, environmental and social aspects.

\section{Step 5: Comparison of scenarios}

In this step the different scenarios are compared. After solving the problem, the GAMS output file is read by VisualSC and the optimal network structure and related data are displayed by the VisualSC interface. The file with the optimized problem can be saved and used at different times to allow the scenarios comparison. In this step the solution computed with the different objectives functions or different network characteristics are compared which eases the selection of the best alternatives.

\section{Decision Support Tool - VisualSC}

VisualSC is an online decision support tool supported by Google maps (geographic information), which allows the creation of optimized supply chain networks (forward, reverse and closed-loop). This DST is a strategic and tactical decision level tool, since the model now available determines the optimal supply chain network (strategic decisions) and defines facilities capacities, production levels, acquisition and distribution plans, collection amounts, among others, for the different supply chain entities (tactical decisions).

To use this DST, the user inserts in the VisualSC all possible locations for the different supply chain entities and defines the echelons (links between entities) and associated data such as capacities, demands, costs, etc. VisualSC collects the required data through a GIS server (as the distances), creating an input file that will be uploaded into optimization software (GAMS/CPLEX). Specific network characteristics of the network might manually introduced/altered by the user. GAMS reads the input file and optimizes the proposed supply chain network. The output generated by GAMS is translated into nodes, which are displayed over the map of VisualSC interface, graphically showing the 
optimal supply chain structure. VisualSC software serves three main objectives: 1) facilitates the development of the network superstructure, 2) builds GAMS inputs files and connects with the optimizer, reducing significantly the required time involved in data collection and 3) visually presents the optimal solution provided by the optimizer. Figure 1 illustrates the architecture of DST VisualSC.

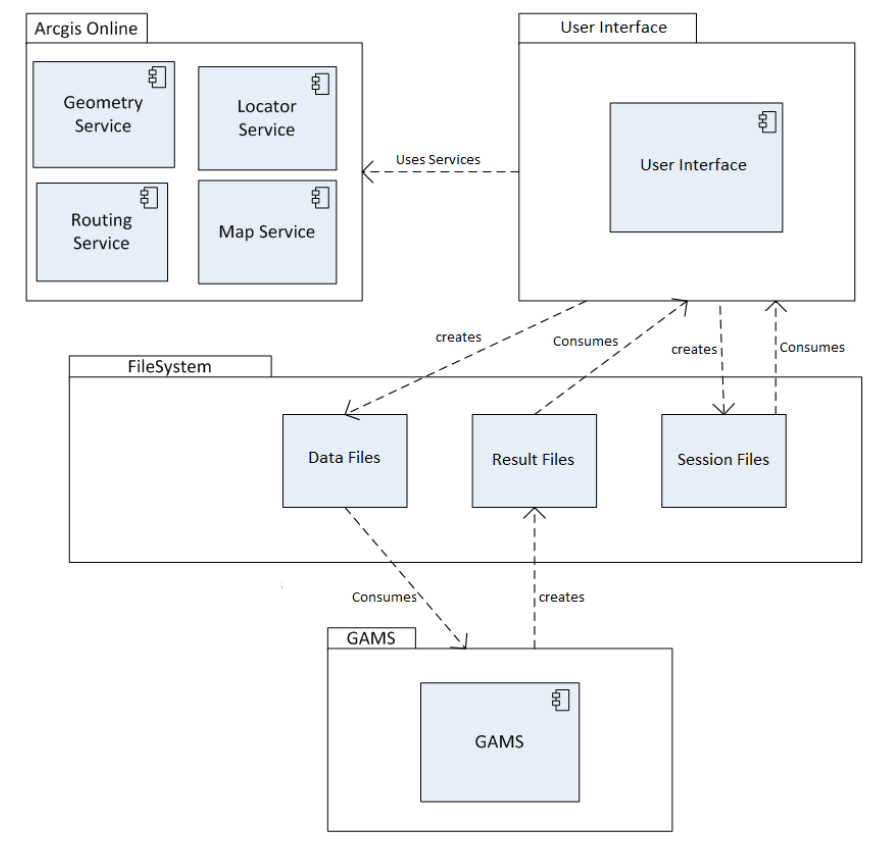

Figure 2: VisualSC Architecture

Figure 2 shows the main modules of the decision-support tool. VisualSC is connected with Arcgis Online, which provides services such as geometry, locator, routing and maps. These are opened access internet services avoiding the complex process of licence acquisition. An intermediate level between the user interface and GAMS/CPLEX provides all the files that connect both levels. These files are available is if a more knowledgeable user wants to access them.

\section{Example}

This case study is related to the strategic planning decisions, in a closed-loop supply chain, for a Portuguese glass company. In order to design its forward and reverse supply chain, the glass company has preselected five locations to build factories, warehouses and sorting centres so that its customers, clustered into 11 Portuguese districts, have their demand and returns fulfilled. When disposed by customers, glass is collected and sent to sorting centres. After quality evaluation and selection process, products are sent back to the factories to be incorporated in the production process or sent to proper disposal. Together with the location decisions, several tactical plans are determined. Namely, the plans for acquisition, production, distribution, and collection amounts are established for two-month periods within a five-year time horizon. Facilities and flows in between are limited in terms of capacities. The number of opened facilities is also 
limited, since the company wants to have at most two factories in operation and at most three warehouses and sorting centres. The company aims at defining the best network structure and operation plan so that the global supply chain cost is minimized. Due to lack of space only two scenarios will be analysed. The first one (case 1) sets all factories costs and capacities as initially estimated by the company. After analysing case 1 solution, the company wanted to investigate if it's preferable to invest in a factory in Lisboa with a larger production capacity and consequently higher opening costs (Case 2).

Using the VisualSC tool, all possible facility and customer locations were introduced, setting for each node its characteristics. The superstructure was then defined as shown in Figure 3. Notice that colours distinguish the supply chain echelons (e.g, flows from factory to warehouses, from warehouses to customer). All input files were then generated and fed to GAMS. After solving the problem, the output was uploaded to VisualSC and plotted over the map of Portugal (see Figure 4)

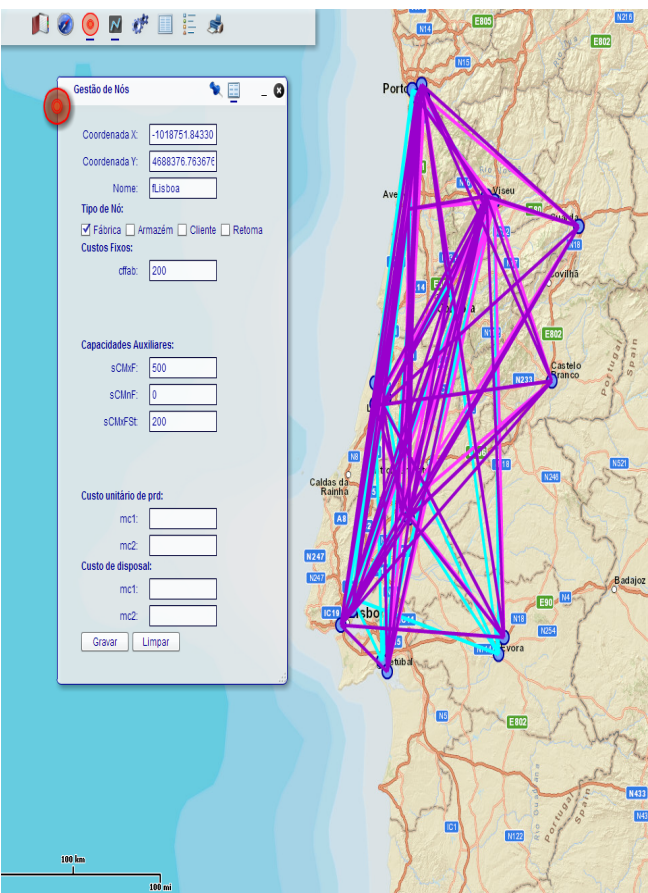

Figure 3: Network superstructure The optimal supply chain has two factories, one located in Lisboa (southern Portugal) and the other in Porto (northern Portugal). Warehouses and sorting centres are opened at the same locations. All customers are served but not fully served. A penalty cost of 2802 monetary units (u.m.) representing about $61 \%$ of the total supply chain cost is incurred. This low quality of service is due to under capacitated factories, therefore the company decided to investigate the consequences of investing in a larger and more expensive factory in Lisboa.

Concerning the structure, case 2 optimal network is the same as case 1 , as the same locations are chosen. As it is possible to verify from Table 1, changes appear at the planning decision level since now it's possible to satisfy almost all customers demand and return (not shown due to lack of space).

The penalty cost represents now less than $10 \%$ of the total cost. Since more products are flowing in the network, therefore the transportation costs increased, more than doubling the initial value.

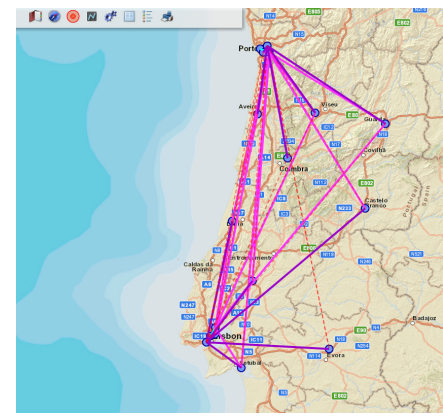

Figure 4: Network optimal structure 
Table 1: Optimal cost structure for cases 1 and 2 (in monetary units)

\begin{tabular}{ccccc}
\hline & $\begin{array}{c}\text { Optimal } \\
\text { cost }\end{array}$ & $\begin{array}{c}\text { Fixed } \\
\text { cost }\end{array}$ & $\begin{array}{c}\text { Transportation } \\
\text { cost }\end{array}$ & $\begin{array}{c}\text { Penalty } \\
\text { cost }\end{array}$ \\
\hline Case 1 & 4578 & 700 & 1075 & 2802 \\
Case 2 & 3608 & 900 & 2379 & 329 \\
\hline
\end{tabular}

However, the trade-off between transportation costs increased and penalty costs

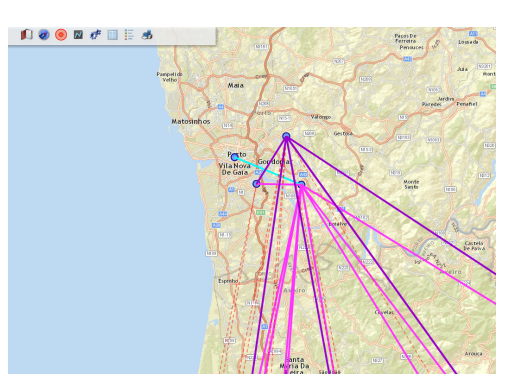

Figure 5: Detail of case 1 optimal supply chain decrease leading to a better supply chain total cost. It decreased from 4578 m.u. to 3608 m.u. which is about $21 \%$ reduction.

Figure 5 zooms the network in Porto region (northern Portugal). It shows with more detail the different facility locations and its connections. Although represented by straight lines, the distance between each node was calculated using Google Maps services, which bring more accuracy to transportation cost calculation. Both cases were solved in GAMS/CPLEX (bluit 23.9.1).

\section{Conclusions}

In this work we proposed a systematic methodology to help companies throughout the difficult process of strategic decision-making. A VisualSC decision-support tool is also developed and presented in this work. The main advantage of this tool is that it allows users (usually companies), which are not familiar with the mathematical models, to optimize their supply chain network, in an easier and user-friendly way. It is important to mention that the proposed methodology integrated with the tool VisualSC is generic and it can be easily extended to include any model. Moreover and as mentioned, some parameters required for the model are calculated automatically by VisualSC, reducing significantly the planning process, and allowing the creation and study of different scenarios.

Currently a second model is being developed to expand VisualSC library. Several improvements have to be made in order to rend it even user-friendlier.

\section{References}

Cai J., Liu X., Xiao Z., Liu J., (2009), Improving supply chain performance management: A systematic approach to analysing iterative KPI accomplishment, Decision Support Systems 46, $512-521$

Guide V.D.R., Van Wassenhove L.N., (2009), OR FORUM - The Evolution of Closed-Loop Supply Chain Research. Operations Research 57, 10-18

Fleischmann M., Beullens P., Bloemhof-Ruwaard J.M., Wassenhove L.N. Van, (2001). The impact of product recovery on logistics network design. Production and Operations Management 10, 156-173

Salema M.I.G., Barbosa-Povoa,A.P., Novais A.Q., (2010), Simultaneous design and planning of supply chains with reverse flows: A generic modelling framework. European Journal of Operional Research 203, 336-349

Seuring S., Muller M., (2008), From a literature review to a conceptual framework for sustainable supply chain management, Journal of Cleaner Production 16, 1699-1710 